\title{
Coral: A Framework based on Social Network Analysis to Support the Startup Ecosystem Management
}

\author{
Rafael Escalfoni (author), Jonice Oliveira (advisor) \\ ${ }^{1}$ PPGI - Universidade Federal do Rio de Janeiro (UFRJ) \\ Av. Athos da Silveira Ramos, 274, Bloco E, Ilha do Fundão \\ Cidade Universitária - Rio de Janeiro, RJ - Brasil \\ rafael.escalfoni@cefet-rj.br, jonice@dcc.ufrj.br
}

\begin{abstract}
Startup ecosystems are business communities continually unfolding where different actors interact in symbiotic activities to create mutual benefits. A smart startup ecosystem demands an understanding of the interests, capabilities, and affinities among members to take harmony and ensure the group's prosperity. The absence of such mechanisms would compromise the innovation process efficiency. Moreover, the environmental imbalance might lead to behaviors harmful to each participant's community to identify convergences and possible partnerships to help develop new business. From this problem, our main contribution is the Coral framework - a social network analysis approach to assist in evaluating relationships in communities. Based on a set of two observational studies in industrial cases, we verified that it is possible to describe the social and material aspects needed to enhance integration and provide greater network efficiency.
\end{abstract}

\section{Introduction}

Innovation depends on a favorable environment to enhance value creation. An entrepreneurial community pursuit an equilibrium between research activities and entrepreneurship to transform ideas from the lab bench to the daily people's lives [Rondani and Colonna 2015]. The business community affects the startups' performance, so understanding how entrepreneurial support organizations provide infrastructure, administrative assistance, and networking is essential to facilitate access to services providers, skilled labor, infrastructure, technology resources, and funding [Stam 2015].

A startup ecosystem is a continuously evolving business community where different entities interact in symbiotic activities to create mutual benefits. Entrepreneurs seek to validate innovative ideas quickly in this creative workspace, converting them into disruptive businesses with low costs. However, it is a complex system composed of several components interacting in a non-simple way, so many individual elements are unpredictably combined and challenging to reproduce in different scenarios. It can affect the decision-making process due to non-trivial emergent behaviors and various social, economic, and technological aspects, such as cultural elements, urban facilities, government support, and other regional groups [Audretsch and Belitski 2017]. Moreover, as the ecosystem becomes more complex as it evolves, members need to manage their relationships to survive and be competitive properly [Basole et al. 2016, Faber et al. 2018]. 
Previous studies point out some features that can be applied to ground our theoretical conjectures. Firstly, we identified that the social network analysis could support the study process in startup ecosystems, depicting their members and relationships (Conjecture 1 - C1) [Motoyama and Waltins 2017]. As a result of the C1, the network analysis use aims to understand the dynamics in which the links are established or extinguished, their main actors, and possible threats and opportunities (Conjecture 2 C2) [Basole et al. 2016, Morris and Schniter 2018]. Such effort enables the identification of actions that can improve partnerships, seeking the interest convergence to increase community resilience (Conjecture 3 - C3) [Tsujimoto et al. 2017]. We considered that the actors and the existing ties could be mapped as a heterogeneous social network, where different members have specific goals in the group.

Another essential component of the study addresses the complementary resources needed for each ecosystem member. West and Bogers (2014) suggest different levels of external resources shared between actors searching for innovation. Each network's participant must know their relevance to their peers and what influences they deploy (Conjecture 4 - C4) [Stam 2015]. Thus, it is necessary to outline partnership strategies and identify how the venture can bring new resources.

Our research problem is related to how ecosystem members interact and seek complementary resources for their business. Although several studies search for a better understanding about how such cooperation is orchestrated [Moore 1993, Prahalad and Ramaswamy 2004, Etzkowitz 2008], collaborative decisions have often been built in an ad hoc manner, based on the intuition and previous knowledge of entrepreneurs and support organization coordinators [Audretsch and Belitski 2017, Escalfoni et al. 2017]. Consequently, there is a need to study the way actors relate and the importance of connectivity to the entrepreneurial community. Therefore, our research question was defined as "How can partnerships in the startup ecosystems be described and represented in order to improve network efficiency and resilience?"

Thus, it was possible to enunciate the research premise as "Managing and monitoring relationships in a startup ecosystem might support opportunity recognition and better resource sharing, enhancing ecosystem efficiency and resilience". This assumption was applied to elaborate the research questions (RQ) that drove the investigation:

RQ1 What are the main aspects of startup ecosystems?

RQ2 What are the types of relationships formed in the startup ecosystems?

RQ3 How to measure aspects of relationships in startup ecosystems?

RQ4 How to represent indicators of relationships in startup ecosystems?

To tackle these issues, we proposed the Coral framework, a set of artifacts to analyze startup ecosystem relationships. The Coral ${ }^{1}$ framework has (i) a model to depict the relationships between startup ecosystem members through different graphs; (ii) a method that defines the process to gather, structure, and instantiate the model; and (iii) a platform to support the method.

\footnotetext{
${ }^{1}$ We call it "Coral" as the marine ecosystem, a habitat of diverse species that suffer directly from external changes.
} 
Wherefore, the main objective of this research is: Understand how relationships influence the efficiency and resilience of startup ecosystems.

This study also has as specific objectives:

- To perform a comprehensive literature review of startup ecosystems, consolidating research in the area of social network analysis to establish the importance of relationships for startup success;

- To propose a startup ecosystem model based on complex network structure in order to highlight socio-technical aspects inherent to entrepreneurial communities;

- To define a method to build a complex network from startup ecosystems, in order to understand the partnership ties;

- To build and test a computational platform to support the method;

- To apply the computational artifact to validate the proposed model.

Our research theme has been gaining prominence in the information systems area, as manifested by the Special Committee on Information Systems of the Brazilian Computer Society (CE-SI / SBC). The paper entitled "Grand Research Challenges in Information Systems in Brazil 2016-2026" brings as its second major challenge "Information Systems and the Open World Challenges", which points out as the main trends for the area the development of techniques and tools that provide greater transparency, collaboration, sharing, and training for organizations and individuals [Araujo et al. 2017]. In the case of startup ecosystems, transparency of governance mechanisms and collaboration are the main drivers of new business.

This work is structured as follows. Section 2 brings the main concepts of a smart startup ecosystem and results of a systematic mapping study to characterize the state-ofthe-art social network analysis applied to startup ecosystems. Then, Section 3 describes the research design and the methodological approach applied in the Coral artifacts development. Next, Section 4 shows the performed Design Science Research cycles, their artifacts, and achievements. After that, Section 5 presents the evaluation process applied in each research phase. Finally, Section 6 concludes with our accomplishments and future works.

\section{Smart Startup Ecosystems}

A startup ecosystem is a set of different agents that promote an entrepreneurial spirit. They follow and support the startup development process, stimulating entrepreneurship, generating innovation, and economic growth [Escalfoni et al. 2018]. They have been transforming urban spaces into vital centers of an innovative economy, capable of making significant social and economic advances [Motoyama and Waltins 2017]. Due to its relevance, cities and regions worldwide have focused effort toward building more intelligent communities, integrating physical, social, and business infrastructure to improve competitiveness. One of the biggest challenges for ecosystem success is understanding the nature of these participants' relationships once actors with different roles and interests form them [Audretsch and Belitski 2017].

Increasingly, entrepreneurial communities have strengthened their intelligence and co-creation collective efforts. Partnerships among main stakeholders are required to share research and innovation assets, such as technology platforms, rising tools, new 
methodologies, and expertise. Thus, it becomes essential to develop tools to better interaction between agents, considering cultural and social norms [Chourabi et al. 2012].

We argue that a smart startup ecosystem has skills to aim for greater awareness, flexibility, transformability, synergy, self-decisiveness, and strategic behavior, according to the smart cities performing model shown by Chourabi et al. (2012). This kind of community must provide tools to gather and integrate diverse data sets, including social media, which can reveal tacit relations about community members [Escalfoni et al. 2019]. A smart startup ecosystem also must have a technology platform to unify data to allow communication among its members' services. It must offer mechanisms to analyze, model, and visualize available network resources to the better operational decisionmaking process.

Thus, a smart startup ecosystem has to make conscious decisions to deal with its social and business needs. For that, it must aggressively deploy emergent information technologies to create a communication infrastructure to integrate its services. Strong ties among people, information, and community elements are requirements to develop competitive and innovative sustainable economic growth [Chourabi et al. 2012].

\subsection{Startup Ecosystem Networking Features}

The complex nature of startup ecosystem relationships is the result of a unique combination of environmental aspects. They are social, cultural, and material factors that influence the discovery and exploitation of opportunities. The cultural elements are related to the sense of confidence and security necessary to establish community collaboration. A friendly culture is concerned with establishing the environmental conditions necessary to stimulate entrepreneurial activity through a climate of greater acceptance of risks [Audretsch and Belitski 2017]. The influence of family and friends is also mentioned as a factor affecting the entrepreneurs' activities [Torres and Souza 2016].

Social capital refers to the benefits obtained or acquired through the community social network. It has a fundamental role in discovering new knowledge about opportunities and technologies, helping new ventures to obtain funding, and influencing new perspectives and entrepreneurial skills. Social capital depends on the established connections and existing culture in the network. These aspects create an atmosphere of trust among the agents, which is a primary condition to encourage the sharing of scarce resources among entrepreneurs, investors, and other entities [Bandera and Thomas 2017].

The material aspects are related to the physical conditions necessary for the startup ecosystem establishment. According to Audretsch and Belitski (2017), the regional infrastructure can influence connectivity and opportunity recognition. The facilities offered by the region can make it more attractive to a higher number of entrepreneurs, local and regional authorities, researchers and academics, educational institutes, and other agents [Motoyama and Waltins 2017].

\subsection{Social Network Analysis}

The social network analysis (SNA) is one of the most established social data techniques. It can aid in searching for knowledge about the interactions between organizations in startup ecosystems by studying the network structural aspects [Olshannikova et al. 2017]. The behavior of certain elements cannot be studied separately due to the influences produced 
by the environment. In such cases, it must consider how connections are formed and their relevance to the problem in question. The startup ecosystems partnerships provide members some resources that otherwise they would not have. As an entrepreneurial community is a set of interdependent organizations, SNA aids in studying its dynamics [Easley and Kleinberg 2010, Neumeyer and Santos 2018].

A social network is an abstraction that allows codifying relationships between pairs of individuals, such as ties of friendship, affinity, shared interests, or commercial relations [Basole et al. 2016]. Some phenomena are occurring in networks that depend fundamentally on their structure. Therefore, the study of the network properties can reveal interaction patterns. SNA can assess partnership coordination levels, interactions intensity, community emergence, connectivity degree, participants' relevance, group influence, and group behavior patterns [Neumeyer and Santos 2018, Ahuja et al. 2012].

\subsection{Social Network Analysis Techniques applied Startup Ecosystems}

The partnerships are a driving force for entrepreneurship, assisting in access to complementary resources and knowledge [West and Bogers 2014]. However, recognizing suitable partners becomes a challenge as the ecosystem evolves, requiring considerable effort to the network management. Studying network properties can reveal patterns of interaction between people and organizations, expanding the understanding of the startup ecosystem dynamics. Thereby, we conducted a systematic mapping study (SMS), producing a deepen compilation of articles concerning current models and metrics of SNA applied in the entrepreneurial community context that brought the insights to improve the Coral Model.

The investigative work verified if such strategies suggest using social network analysis techniques to form and maintain enduring and beneficial partnerships, enumerating the existing challenges in the area. First, the SMS protocol was developed with three other researchers' cooperation to mitigate potential research biases. It was established a procedure for identifying, selecting, and evaluating relevant work to describe which partnership management methods are applied to startup ecosystems and what type of SNA metrics are implemented to map and evaluate relationships. The literature review used the same search strategy on different bases to ensure consistency and focus on the work scope. Then, to perform the searches, we chose the most relevant digital libraries in the business and computing areas: ACM Digital Library, IEEE Digital Library, Scopus, Science Direct, Social Science Citation Index (Web of Science), and Springer Link. The bibliographical review only considered peer-reviewed English articles published in journals and conferences between 1993 - the year in which the term "business ecosystem" was coined - to 2018, considering accepted papers for publication in 2019. Figure 1 illustrates the performed SMS workflow.

The literature review depicted the relationships, the main aspects considered by participants to seek partners, and the different approaches used to manage network resources, as showed in Table 1. We demonstrated the importance of models based on social network analysis to manage and support interactions in different communities through current research. The investigation showed that it is possible to define a toolset to assist in business network management using models based on graph theory. 


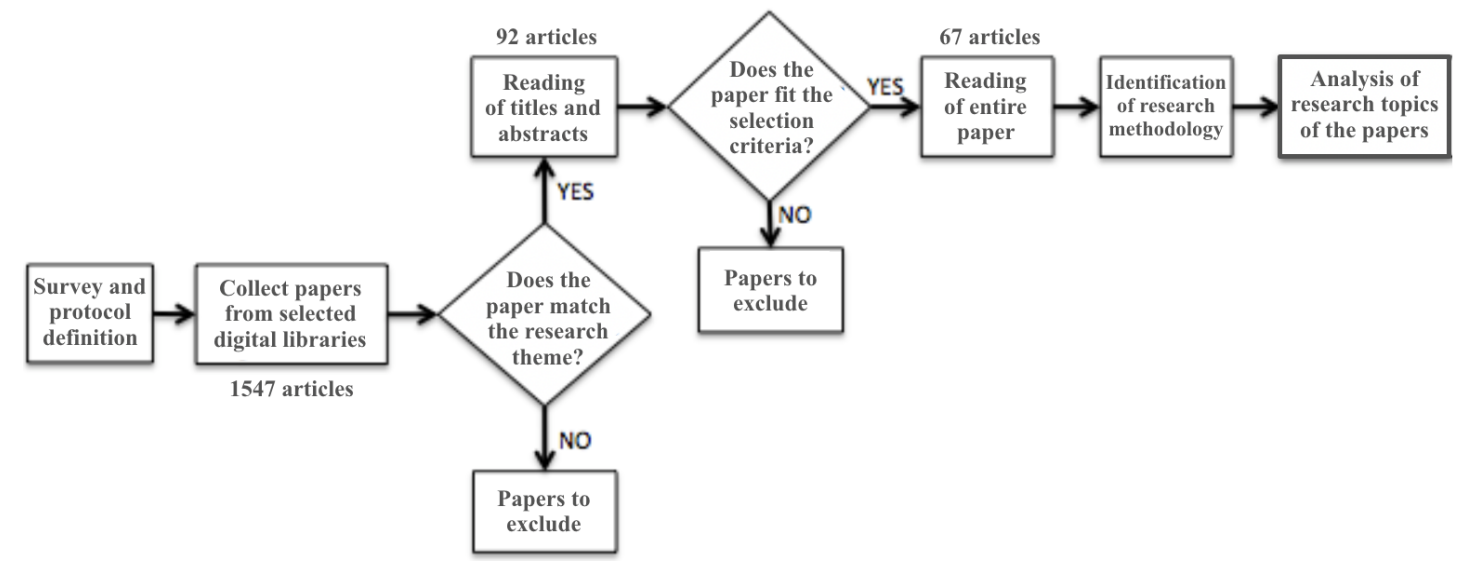

Figure 1. Systematic Mapping Study execution

Table 1. Social Network Analysis Metrics for Evaluating Startup Ecosystems

\begin{tabular}{|l|l|l|}
\hline \multicolumn{1}{|c|}{$\begin{array}{c}\text { Type of } \\
\text { Relationship }\end{array}$} & \multicolumn{1}{|c|}{ Indicator } & \multicolumn{1}{c|}{ References } \\
\hline $\begin{array}{l}\text { Among } \\
\text { Entrepreneurs }\end{array}$ & $\begin{array}{l}\text { Degree, Density, Modularity, } \\
\text { Centrality (Degree, Betweenness, } \\
\text { Eigenvector, Closeness), K-Step } \\
\text { Reachability }\end{array}$ & $\begin{array}{l}\text { Hoang and Antoncic (2003), Ahuja, } \\
\text { Soda and Zaheer (2012), Teigland et } \\
\text { al. (2012), Kathan, Füller and Hutter } \\
\text { (2013), Neumeyer and Santos (2018) }\end{array}$ \\
\hline Among ESOs & $\begin{array}{l}\text { Degree, Density, Cluster Coefficient, } \\
\text { Centrality (Degree, Closeness) }\end{array}$ & $\begin{array}{l}\text { Chen and Lin (2017), Escalfoni et al. } \\
\text { (2018) }\end{array}$ \\
\hline Entrepreneur - \\
ESO & $\begin{array}{l}\text { Degree, Degree Distribution, Density, } \\
\text { Average Shortest Path Length, } \\
\text { Centrality (Degree, Betweenness, } \\
\text { Closeness), Clique Analyis }\end{array}$ & $\begin{array}{l}\text { Phelps, Heidl and Wadhwa (2012), } \\
\text { Yoon et al. (2015), Fuster et al. } \\
\text { (2019), Chen and Lin (2017), Song et } \\
\text { al. (2018) }\end{array}$ \\
\hline $\begin{array}{l}\text { Entrepreneur - } \\
\text { Investor }\end{array}$ & $\begin{array}{l}\text { Degree, Density, Centrality (Degree), } \\
\text { Shortest Path, Common Neighbors }\end{array}$ & $\begin{array}{l}\text { Eugene and Yuan (2012), Clarysse } \\
\text { et al. (2014) }\end{array}$ \\
\hline ESO - Investor & Degree, Centrality Betweenness & Battistella et al. (2013) \\
\hline \multirow{2}{*}{ Ecosystem } & $\begin{array}{l}\text { Degree, Distance, Shortest Path } \\
\text { Length, Size, Density, Centrality } \\
\text { (Degree, Betweenness, Eigenvector, } \\
\text { Closeness), Cluster Coefficient }\end{array}$ & $\begin{array}{l}\text { Battistella et al. (2013), Basole et al. } \\
\text { (2014), Basole et al. (2015), Faber et } \\
\text { al. (2018), Mamede, Escalfoni and } \\
\text { Oliveira (2018) }\end{array}$ \\
\hline
\end{tabular}

\section{Research Design}

The planning of the Coral approach was based on a series of iterations designed in a problem-solving cycle according to Design Science Research (DSR). The DSR grounds and operationalizes the research conduction using the epistemological basis of design science. Its approach is problem-oriented, so the method steps aim to understand the needs, build, and evaluate artifacts that allow transforming situations, changing their conditions to better or desirable states [Hevner et al. 2004]. Its strategy inspired the enumerated research questions, building and evaluating research activities to provide the required artifacts to characterize and analyze startup ecosystems' social and technological aspects, as illustrated in Figure 2.

The first DSR cycle intended for answering the RQ1 - What are the main aspects of the startup ecosystems?. We have contextualized the innovation background and then executed a literature review about entrepreneurial communities, focused on the fundamental concepts, actors, environmental influences to the group progressing. As a result, the study brought some findings on the importance of social, cultural, and technological aspects. Moreover, we identified ecosystem maturity models and resilience aspects that have driven out a resilience model for the startup ecosystems. We also 
After identifying a set of useful metrics for assessing relationships in startup ecosystems, the RQ4 - How to represent indicators of relationships in startup ecosystems? guided the fourth DSR cycle. Thus, a framework was developed to systematize conceptual model utilization. The Coral Framework includes a protocol for defining data sources and an outlined method to collect raw data, giving it meaning, building graphs, and apply the Coral model. At this point, a feasibility study was conducted in the Rio de Janeiro state startup ecosystem, as reported in [Mamede et al. 2018].

To assist in our research activities, we have developed the Coral Platform, which consists of a web-based integrated system that helps the Coral framework, executing an automatic gathering of open data, including social media, structures the information, and makes it available to queries through graphs previously defined in a protocol. The Coral platform was tested through a case study to assess existing connections among engineering startups of a local community [Escalfoni et al. 2020a]. The study gave feedback to improve the Coral approach, such as new metrics recommendations, layout, and graphs adjustments [Escalfoni et al. 2020b].

The research achievements provided a set of lessons learned about enhanced startup ecosystems and a toolkit to deal with heterogeneous open data sources to characterize entrepreneurial communities supporting decision-making processes and enhancing collaboration among their participants. We believe that it can be generalizable to other types of groups, such as research groups and cooperatives.

\section{Coral Framework}

A framework is a body of knowledge that supports propositions and constructs based on emerging theory. It consists of an adaptable artifact that can be revised and extended to new investigation processes [Gomes et al. 2016]. Our approach comprises extensible modules to allow adaptation according to data availability and the intended analyses. In this way, the Coral framework aids in collecting, treating, and analyzing an observed entrepreneurial community's social data. The different online social media and data sets that provide information about the ecosystem require specific web crawlers for the data collection phase. Thereby, we defined a procedure to define gathering strategies. Once collected, the data should be classified and structured to be consulted, evaluated, and processed. Next, the structured data are submitted to modules to characterize entities, relationships, and business ties for further investigations.

To assist in the formulation of the modules, we propose an architecture that establishes the nature of the different framework component modules, as shown in Figure 3. According to the scheme, the collected data goes through a structured curation process to ensure the data quality according to the specified data set. Then the data is used to characterize the organizations and their relationships. The entities module allows describing the different entrepreneurial community participants. Several dimensions can be applied to describe the organizations, according to the availability of data and necessity, such as geolocation, sector, skills, reputation, interests, and portfolio of each ecosystem member. In turn, the relationships module investigates the ties between entities under the social and material aspects. It might represent different community associations, being used to expose resources available to a specific group, point out the most prominent 


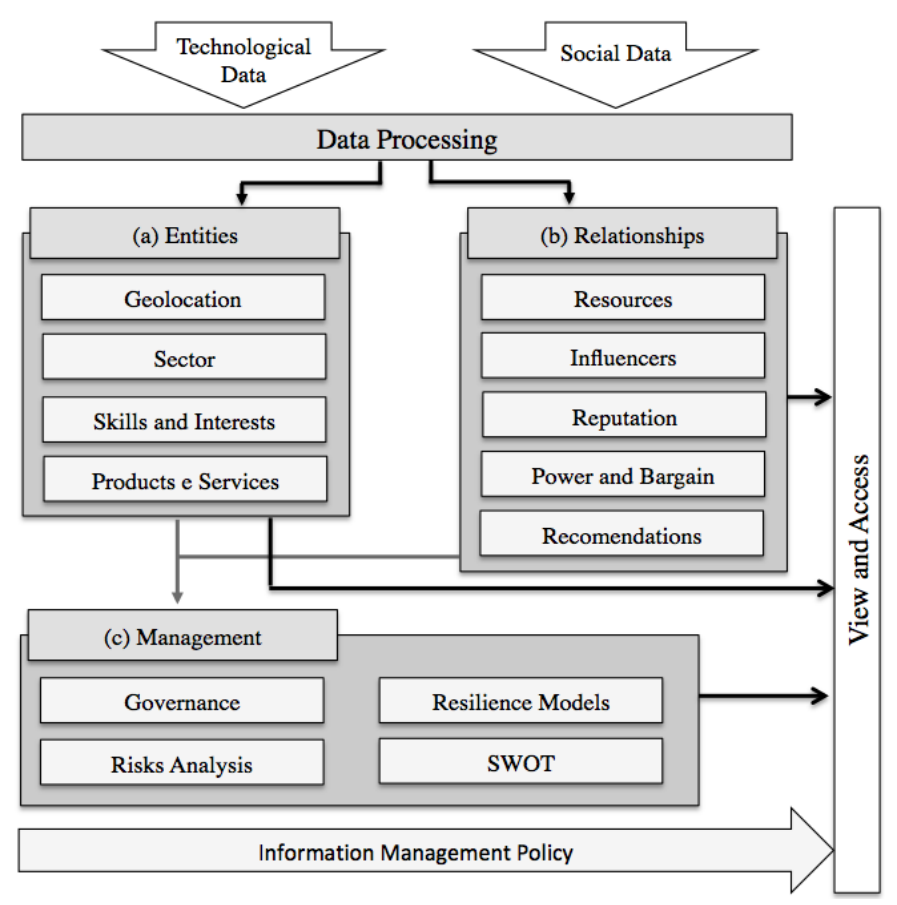

Figure 3. An Overview of Coral Framework

members, recommendations, and each participant's reputation. The management module aims to analyze elements of the community as a whole. It is possible to support analysis for different ecosystem aspects by combining some Coral model indicators. How organizations articulate their networks can reveal if the governance strategies with their legal boundaries, agreements, and rights protections are adequate [Stam 2015].

Similarly, a temporal analysis of ecosystem performance can expose its resilience [Woods 2015]. Even the ecosystem smartness can be measured according to its innovative skills and produced economic growth [Motoyama and Waltins 2017]. The visualization and access module seeks to define relevant information for each participant profile, be it an entrepreneur, entrepreneurial support organizations, or investors group. The information availability follows an information management policy agreed upon among the startup ecosystem participants.

Our approach provides acknowledging specialized data sets recommended by experts and open databases available on the Internet as data sources, such as online social media platforms and company outreach channels. For that, the Coral framework has a method based on four stages: (1) Community Platform Identification, when the most relevant data sets for collecting are defined in a search protocol; (2) Gathering of Business Data and Redundancy Elimination, step in which the web crawlers and others search engines are developed. The collected database is organized and structured through taxonomies and clustering algorithms; (3) Data Integration and Network Modeling, phase to unify the different databases and to specify the graphs in order to aim the knowledge identification; and (4) Startup Ecosystem Analysis and Assessment, phase to submit the graphs to SNA metrics, patterns, and behaviors observation, depicted in Figure 4.

The Coral platform guarantees an automatic gathering process, independent of the stakeholders' engagement to support the execution. It can avoid resistances that 


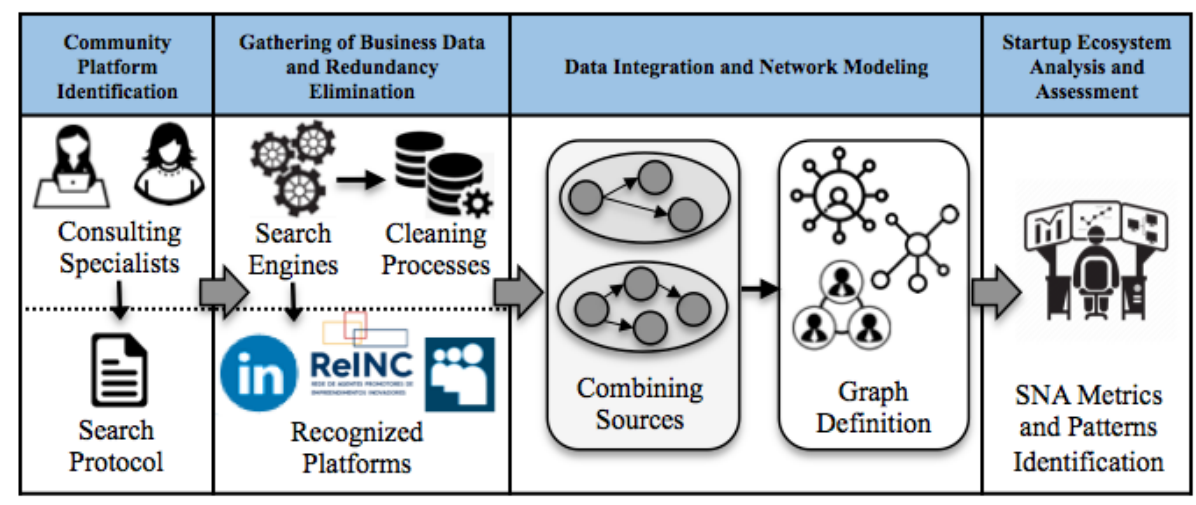

Figure 4. Coral Method

could compromise the data accuracy or could cause a lack of information. The big data collected from the intelligent environment can play an essential role in describing business elements and relationships. It could also help identify hidden patterns and correlations [Escalfoni et al. 2020a]. Information analysis can provide insights to assist decisionmaking processes and improve, among other things, sustainability, resilience, and governance of smart cities and, therefore, startup ecosystems [Olshannikova et al. 2017].

Through the Coral model, it is possible to identify some important social aspects that can influence partnership creation and maintenance. The participant's interests define the specific interfaces for each type of startup ecosystem participant. Then, the Coral platform is adjusted by the Coral model to define specific measures for analysis. For example, entrepreneurs explore the full potential of the community to deal with their resource constraints. Thus, the tool is set up to provide information about resources, strategic partners, and the environment. By understanding who the community members are, the entrepreneurial team can better plan their actions by identifying their key partners, suppliers, dependencies, and everything essential to the success of their project [Rondani and Colonna 2015].

The system currently has three dynamic graphs that identify aspects of participants, competencies, and reputation of each one, as illustrated in Figure 5. The tool provides a graph and the most important related SNA metrics. It also implements filter engines to specify the type of venture (projects, startups, newly created businesses, enterprises, or ecosystem builders), local community, and sectors. Finally, the system offers a report detailing the result of each graph.

\section{Constructs Evaluation}

To assess the different DSR cycles developed throughout the research, we carried out evaluation and learning steps to improve the constructs developed and consolidate the research contributions. We validated our first premises through a literature review, which helped us improve the understanding of social and cultural ties in entrepreneurial communities.

The indicators for studying entrepreneurial relationships identified previously in the literature review were examined in an exploratory study in two entrepreneurial communities in the Rio de Janeiro state. For this, we conducted interviews with specialists seeking to understand partnership networks' role in business development. The results 


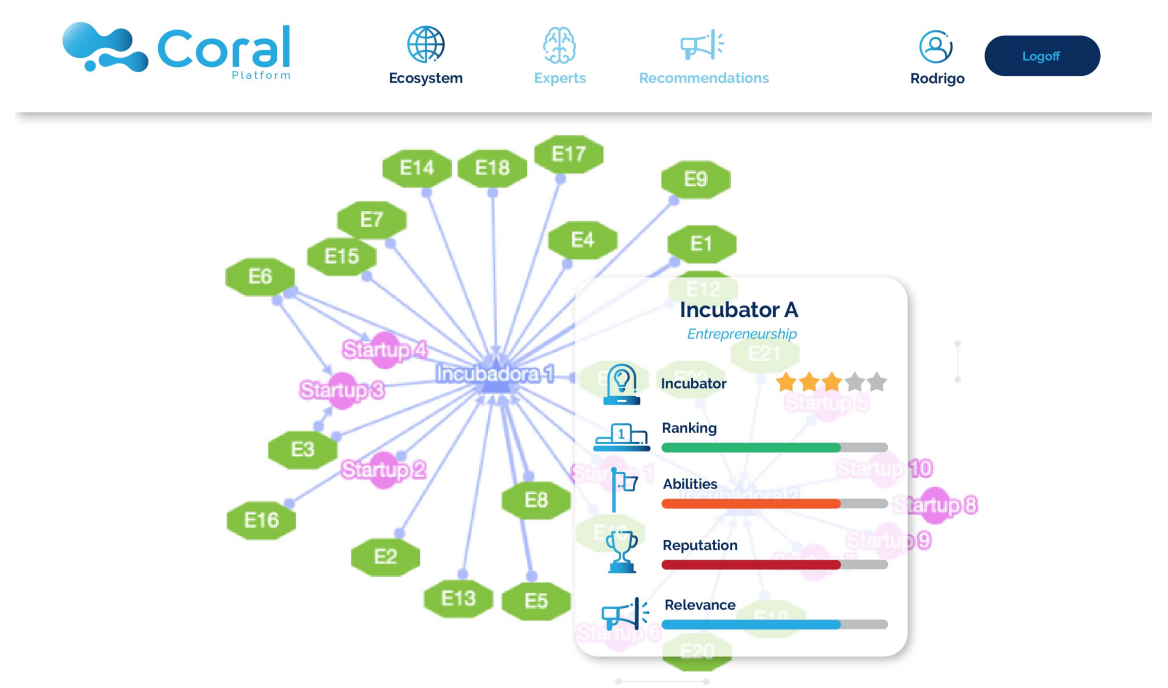

Figure 5. Coral Platform - Participants Graph [Escalfoni et al. 2020a]

brought evidence that the critical ties that make up the construct developed in the second DSR cycle - "indicators for evaluating entrepreneurial relationships" were contemplated in the studied communities. As contributions to this cycle, we highlight recognizing the importance of social relations for launching startups.

Then, in the third DSR cycle, we executed the Coral model in a community of practices formed up of technology business incubator managers aiming to verify its applicability. The results showed that the model might assist in the perception of intrinsic characteristics of the startup ecosystems. Some notes of improvements and future work were also identified, such as using heterogeneous databases to characterize the participants, incorporated in the Coral Framework proposal.

During the fourth DSR cycle, we aimed to assess the Coral Framework feasibility. We mapped the regional startup ecosystem of Rio de Janeiro, highlighting the member organizations' aspects that influence a professional and innovative community. The experiment sought to depict the entire entrepreneurial ecosystem formed by incubators and technology parks linked to major universities and research centers in the region and their partners. For that, we applied our construct, collecting diverse available data on the Internet from institutional and social media platforms seeking information to characterize the community. Then, we compared the group profiles with graduated startups, which become successful companies, revealing each community's best practices and areas. The social network analysis applied broadened the understanding of tacit social ties by gathering diverse data sources, identifying entrepreneurs, incubators, and technology parks' profiles. Part of this research was published in [Mamede et al. 2018].

Our computational artifact was also tested in a study about the incubator manager's perception of the partnership network's importance to boost the nascent business, as presented in Escalfoni et al. (2020b). The Coral platform supported incubators' activities in the local community improvements, depicting incubator managers' indicators. Unfortunately, the participants have had difficulty interpreting the tool's report, so, to overcome the problem, we reformulated its interface, as published in 
Escalfoni et al. (2020a).

\section{Conclusion}

This thesis supports the study of startup ecosystems, highlighting the theme relevance to the research challenges of the information systems community [Araujo et al. 2017]. The contributions become apparent through the interpersonal ties analysis and their consequences in the rise of nascent companies, in the improvement of processes for the entrepreneurial ecosystems' management, and the design of computational tools for visualization and metrics' application of such spaces. For that, we analyzed communities as social networks with interdependent and coevolutionary ties from a perspective of complex systems, where different actors seek to innovate. As it is a highly collaborative and dynamic environment, it is essential to study partnerships to ensure harmony in the network. Our approach implements mechanisms to monitor shared resources and the most relevant social and technical aspects for each business ecosystem.

The research was framed with DSR, a methodological approach that follows an iterative process through interviews and literature reviews, analysis and diagnosis, artifact development, experiments, and learning assessment. For each cycle, we defined an evolutionary research question that complemented the others.

\subsection{Contributions}

The doctoral research produced scientific and technological contributions to improve startup ecosystems' integration by identifying the socio-technical aspects in studied communities. Our validation stages provided an effort to expand research boundaries in Rio de Janeiro, involving incubators and technology parks from the entire state, and the CEFET/RJ and the Nova Friburgo local government, a medium city from the countryside mountain region. As a result, we stimulated these communities to be aware of the importance of the startup ecosystem networks, make them more open-minded to actions to improve the partnership management based on our artifacts.

The research achievements led to an ongoing master's project under my coadvising, which aims to identify the influence of networking on startups' performance [Mamede 2021], and inspired a final project in Information System [Guinelli 2018]. During the project, six papers were published in prestigious venues, as presented through this article. Besides, we were awarded the IEEE Young Investigator Training Program (YITP) [Escalfoni et al. 2019], which result in an academic visit to the University of Brescia, Italy, to research SNA techniques in the knowledge management domain.

The presentation of the developed components throughout the thesis as interdependent elements simplifies reuse in other research. In this way, this work provides a set of artifacts instead of an aggregate construct. The most notable scientific contributions are:

- Literature review on ecosystems: it brings a comprehensive study of business communities' that presents the evolution of innovation models that justifies the rise of lean startups as drivers of creativity and then discusses the differences between business, innovation, and entrepreneurial ecosystems. Finally, we detailed the main aspects of startup ecosystems, as main actors, environmental influences, and development stages. 
- Resilience study: it proposes a resilience model for startup ecosystems, addressing the community's fundamental features to respond appropriately to changes that may occur.

- Ecosystem smartness: it enumerates the basic requirements for an entrepreneurial region to become a smart startup ecosystem. The concepts of big social data were extended in a strategic sense, discussing how network aspects can improve a smart city's decision-making process. Finally, we introduced indicators that can assist in this process and different factors that must be considered, such as community resilience, types of relationships, and factors that influence communities' development.

- Literature review on startup ecosystem relationships: A body of knowledge on different types of startup ecosystem relationships based on a literature review and a survey with specialists of two different technology business incubators. We identified the nature of the most common interactions and formulated questions about shared resource management processes, leading to the Coral Model proposition.

- Social behaviors identification: Identification and definition of the importance of pertinent social aspects, such as trust ties, similarity, homophily, power relations, and ecological practices such as competition, predation, parasitism, symbiosis, and commensalism.

- SMS on SNA metrics for startup ecosystems: The primary concepts of smart cities were presented, highlighting the importance of startup ecosystems to improve urban spaces' efficiency in service delivery and economic and social development. Then, the basic requirements for an entrepreneurial region to become a smart startup ecosystem were enumerated. Besides, we have extended the concepts of big social data in a strategic sense, discussing how network aspects can improve a smart city's decision-making process. Finally, we introduced indicators that can assist in this process and different factors that must be considered, such as community resilience, types of relationships, and factors that influence communities' development.

- SNA indicators for startup ecosystems: We surveyed information needs for each group of startup ecosystem actors. We then define a set of indicators to be developed to support such participants. These are suggestions for community views and applicable SNA metrics.

Concerning technological contributions, we highlight the consolidated artifacts after the research accomplishment. Among them, the following stand out:

- Coral Model: An SNA-based approach with a set of indicators to map and depict different relationships and interaction behaviors in startup ecosystems, based on the conducted SMS findings.

- Coral Framework: Our integrated environment establishes a guideline for data collection, structuring, and curation, which provides a data set for the Coral Model accomplishment.

- Coral Platform: a web tool that provides an interface for visualizing graphs and tables with the Coral model indicators that enables business experts to conduct their community analysis.

- RJ Startup Community Data Set: We have built a rich data set of the entrepreneurial community from Rio de Janeiro state for further studies. 


\subsection{Limitation}

This doctoral research has some theoretical and practical limitations. Although we performed a detailed literature review, including a systematic mapping study, the research team's lack of business experts might have compromised the proposed artifacts' quality. The experiments' feedback pointed out the current metrics are difficult to understand by people who do not know the fundamentals of social network analysis. We frequently interacted with the local specialists to tackle these threats, but much work remains to be done to improve the indicators and propose new ones.

Regarding the practical constraints, it is noteworthy that the experiments were all conducted in Brazil - in the Rio de Janeiro state entrepreneurial communities. Thus, our approach might only reflect existing needs and problems in the national scenario. The sample size and interviewee profile corroborate the experimental study limitation. We seek to reduce this risk by researching similar work in international communities in the literature, and there is strong evidence that the challenges addressed in this thesis are global. However, we recommend reproducing experiments with the Coral Framework in other communities to analyze artifacts' applicability.

Furthermore, we restricted the research scope to supporting the ecosystem analysis under the managers' profile of support organizations - incubators and technology parks. Currently, there is an ongoing Master's research in which an instantiation of the Coral Framework is being done to support the entrepreneur profile [Mamede 2021]. The results would support our hypothesis that the proposal can meet diverse user profiles in startup ecosystems.

\subsection{Future Work}

There are many possible enhancements and advancements for the research. Several projects can be developed to expand the different parts of the Coral Framework. For example, the Coral architecture has management modules instantiable for different participants in the entrepreneurial community. The detailing and development of such components can assist in resource monitoring and management tasks, improve resilience and planning mechanisms, furnishing the network with greater efficiency.

Moreover, creating inference mechanisms to identify network relationships would significantly reduce data analysis work. Developing adaptive ontologies that could serve entrepreneurial communities would be a stalwart supporter of big data applications, for example. Another helpful functionality would be developing relationship recommendation methods to aid different profiles, especially entrepreneurs, analyze resource availability by better planning and managing their partner networks.

Startup ecosystems perform a predominant role in smart cities. Even though we spotted some critical issues about this aspect, there is a great potential to start research exploring the impacts of big social data technologies on developing a creative economy and knowledge-based society. There are also complex implications in startup ecosystems that must be deepened to create resilience models that reflect the observed communities' reality. A cross-sectional study of an entrepreneurial community could bring original contributions to understanding the evolution of the relationships. 


\section{Acknowledgment}

We thank CEFET/RJ for supporting this research. We also thank CAPES, CNPq, and FAPERJ (Brazil) for their financial support to the research group.

\section{References}

Ahuja, G., Soda, G., and Zaheer, A. (2012). The genesis and dynamics of organizational networks. Organization Science, 23(2):434-448.

Araujo, R. M., Boscaroli, C., and Maciel, R. S. P. (2017). Information systems and the open world challenges. In I GranDSI-BR: Grand Research Challenges in IS in Brazil 2016-2026, Porto Alegre. Special Committee on Information Systems (CE-SI). Brazilian Computer Society (SBC).

Audretsch, D. B. and Belitski, M. (2017). Entrepreneurial ecosystems in cities: establishing the framework conditions. Technology Transfer, 42:1030-1051.

Bandera, C. and Thomas, E. (2017). Startup incubators and the role of social capital. In Technology \& Engineering Management Conference, Kansas, USA. IEEE.

Basole, R. C., Huhtamaki, J., Still, K., and Russel, M. G. (2016). Visual decision support for business ecosystem analysis. Expert Systems With Applications, 65:271-282.

Chourabi, H., Nam, T., Walker, S., Gil-Garcia, J. R., Mellouli, S., Nahon, K., Pardo, T. A., and Scholl, H. J. (2012). Understanding smart cities: An integrative framework. In 45th International Conference on System Sciences, pages 2289-2297, Hawaii. IEEE.

Easley, D. and Kleinberg, J. (2010). Networks, Crowds, and Markets: Reasoning about a Highly Connected World. Cambridge University Press, Cambridge.

Escalfoni, R., França, T. C., Irineu, M. A. S., Vivacqua, A. S., and Oliveira, J. (2018). Um método para apoiar a identificação de interesses entre participantes de ecossistemas de startups. In Brazilian Symposium on Information Systems, Caxias do Sul. ACM.

Escalfoni, R., Irineu, M. A. S., and Oliveira, J. (2017). Impacto das redes de negócio para startups: Um estudo empírico na ietec/cefet-rj. In EISI - Brazilian Symposium on Information Systems, pages 601-604, Lavras. SBC.

Escalfoni, R., Marinho, L., Lacerda, C., Oliveira, R., Mamede, K., Silva, M. F., and Oliveira, J. (2020a). Coral platform: The smart startup ecosystem tool. In EISI Brazilian Symposium on Information Systems, São Bernardo do Campo. ACM.

Escalfoni, R., Silva, M., and Oliveira, J. (2019). Coral framework - a big social data approach to boost startup ecosystem. In Int. Conf. on Artificial Intelligence and Knowledge Engineering, Cagliary, Italy. IEEE.

Escalfoni, R., Silva, M. F., and Oliveira, J. (2020b). Visualizing social relations in startup ecosystems using the coral platform. In Brazilian Symposium on Information Systems, S. B. do Campo. ACM.

Etzkowitz, H. (2008). The triple helix - university-industry-government: inovation in action. Taylor \& Francis Group, New York.

Faber, A., Hernanderz-Mendez, A., Rehm, S.-V., and Matthes, F. (2018). An agile framework for modeling smart city business ecosystems. In International Conference on Enterprise Information Systems, volume 2, pages 39-50, Funchal. ACM. 
Gomes, L., Facin, A., Salerno, M., and Ikenami, R. (2016). Unpacking the innovation ecosystem construct: evolution, gaps and trends. T. F. \& S. Change, 136:30-48.

Guinelli, J. (2018). Belize: Uma ferramenta de suporte na identificação de parceiros em ecossistemas de startups. Undergratuated project, CEFET/RJ, Nova Friburgo.

Hevner, A. R., Marchand, S. T., Parkand, J., and Ram, S. (2004). Design science in information systems research. MIS Quarterly, 28(1):75-105.

Mamede, K. (2021). Um metodo de apoio a analise de redes empreendedoras de ecossistemas de startups. Dissertation, UFRJ, Rio de Janeiro.

Mamede, K., Escalfoni, R., and Oliveira, J. (2018). A method to identify social and technological features in startup ecosystems: a case study of rio de janeiro state. In Workshop on Big Social Data and Urban Computing, Rio de Janeiro. VLDB.

Moore, J. (1993). Predators and prey: The new ecology of competition. HBR, 71:75-86.

Morris, J. J. and Schniter, E. (2018). Black queen markets: commensalism, dependency, and the evolution of cooperative specialization in human society. Bioecon, 20:69-105.

Motoyama, Y. and Waltins, K. (2017). Examining the connections within the startup ecosystem: A case study of st. louis. Entrepreneurship Research Journal, 7(1).

Neumeyer, X. and Santos, S. C. (2018). Sustainable business models, venture typologies, and entrepreneurial ecosystems: A social network perspective. J C. Production, 172:4565-4579.

Olshannikova, E., Olsson, T., Huhtamaki, J., and Karkkainen, H. (2017). Conceptualizing big social data. J. Big Data, 4(3).

Prahalad, C. K. and Ramaswamy, V. (2004). The future of competition: Co-creating unique value with customers. Harvard Business Press, Boston.

Rondani, B. and Colonna, C. (2015). Open startups: Connecting startups to corporate innovation strategies. In XXXIX Encontro da ANPAD, Belo Horizonte. ANPAD.

Spagnoletti, P., Resca, A., and Sæbø, Ø. (2015). Design for social media engagement: Insights from elderly care assistance. Strategic Information Systems, 24(2):128-145.

Stam, E. (2015). Entrepreneurial ecosystems and regional policy: a sympathetic critique. European Planning Studies, 23(9):1759-1769.

Torres, N. N. J. and Souza, C. R. B. (2016). Uma revisão da literatura sobre ecossistemas de startups de tecnologia. In Brazilian Symposium on Information Systems, pages 385392, Florianópolis. SBC.

Tsujimoto, M., Kajikawa, Y., Tomita, J., and Matsumoto, Y. (2017). A review of the ecosystem concept - toward coherent ecosystem design. Tech. F. \& Social Change.

West, J. and Bogers, M. (2014). Leveraging external sources of innovation: A review of research of open innovation. J. Product Innovation Management, 31(4):814-831.

Woods, D. (2015). Four concepts for resilience and the implications for the future of resilience engineering. Reliability Engineering and System Safety, 141:5-9. 